Case Report

\title{
Applying neuromuscular techniques in the orthodontic setting
}

\author{
Savastano, Fabio * \\ * International College of Neuromuscular Orthodontics and Gnathology (ICNOG), Albenga, Italy
}

\begin{abstract}
Introduction: Neuromuscular orthodontic techniques that investigate the interrelatedness of the muscles, bones, joints and teeth involved in oral function, hold promise for the treatment of a spectrum of oral disorders. Indeed, such technologies will help patients receive a more targeted level of care. Here we present a case report concerning an 11-year-old boy who underwent orthodontic treatment for recurrent pain of the right temporomandibular joint (TMJ), misaligned teeth, and irregular clicking noises (associated with the right TMJ) during mouth opening. The basic principles of the use of mandibular tracking, surface electromyography, and transcutaneous electrical nerve stimulation (TENS) to diagnose malocclusions and determine the cranio-mandibular relationship are outlined.

Case presentation: Pre-treatment status, progress, post-treatment status and 8-year follow-up data are shown.

Conclusion: As neuromuscular orthodontics can provide detailed functional analyses through a combination of technologies, the clinician is better placed to evaluate the needs of the patient and deliver treatment. Further deployment of such techniques should, therefore, be encouraged to increase orthodontic health and practice.
\end{abstract}

Savastano F. Applying neuromuscular techniques in the orthodontic setting. South Eur J Orthod Dentofac Res. 2017;4(2):31-42.

Submitted: April 26, 2017; Revised: September 2, 2017; Published: October 15, 2017

\section{INTRODUCTION}

\section{Neuromuscular dentistry and orthodontics}

Nowadays, neuromuscular dentistry (ND) is practiced by many dentists around the world. ND can be defined as a diagnostic and treatment procedure in which the triad of the teeth, temporomandibular joint (TMJ), and masticatory muscles, is considered clinically. Dr. Bernard Jankelson introduced this methodology in the late $1970 \mathrm{~s}^{1-7}$, although there has been limited application of neuromuscular dentistry for orthodontic purposes. Such combinatorial approaches not only increase the amount of physiological data that can be obtained, but also allow for relationships between different systems to be inferred; i.e., how a defect in the mandibular ramen influences joint congruity and masticatory function.

ND involves multiple quantitative and qualitative measurements and procedures, including mandibular tracking,

\section{Corresponding Author:}

Savastano, Fabio

Piazza E. Berlinguer 14, Albenga (SV), Italy

e-mail:Savastano@ICNOG.com surface electromyography, and transcutaneous electrical nerve stimulation (TENS).

Neuromuscular diagnoses rely upon assessments at the mandibular rest (voluntary, head upright) position. Mandibular tracking systems have proved useful in assessing the characteristics of mandibular movements in both normal and pathologic occlusions. ${ }^{8-15}$ Surface electromyography is also a well-established procedure, and many dentists use it routinely. ${ }^{16-19}$ On the other hand, the use of TENS occurs relatively rarely in the dental clinic. Alongside financial barriers to the acquisition and use of the technology, the infrequent use of TENS may be due to a lack of information within dental communities about the importance of muscle relaxation, and the consequential changes in the spatial relationship between the cranium and the mandible. ${ }^{20-23}$ For example, the use of TENS in growing patients can provide valuable information allowing for the avoidance of procedures that could put occlusion stability at risk and increase the chance of TMJ complications. As the basic principles of neuromuscular dentistry have been only partially applied to orthodontics, we here aim to provide a primary introduction to general diagnostic and treatment guidelines for neuromuscular orthodontics $(\mathrm{NO})$ and illustrate their use in the clinical setting. 
Overview of mandibular tracking, electromyography, and TENS procedures

During a complete functional analysis, the first step is to determine the physiological habitual movements of the mandible and muscles. These movements are the result of a muscle engram continuously generated in the CNS that fine tunes mandibular closing in order to match repetitively a precise centric occlusion (CO). This "muscle memory" is the result of the physiologic demands of dental occlusion, and the implications it has on our social and biological function. ${ }^{24,25}$ Analysis entails performing mandibular tracking during opening and closing of the lower jaw and during swallowing. Surface electromyography is then performed during mandibular rest, clenching, and movement. Thus, this first step involves evaluating the mandibular postural muscle function that is conditioned by occlusion (accommodation). ${ }^{25}$

Periodontal stimuli sent to the CNS provide an active motor output signal as part of a feedback mechanism leading to the determination of the mandibular rest position. This habitual rest position is the most functional position for the patient's occlusion, as it minimises the energy required to move to a centric occlusion (CO) ${ }^{25}$ In other words, the CNS controls and guides the mandible to a position in which swallowing (a semi-autonomic physiological process) uses the least amount of energy possible given the physiological conformation present. The inter-occlusal space, or freeway space, is between 2 and $4 \mathrm{~mm}$ at length in the majority of the general population. ${ }^{23,26-28}$ The CNS receives several afferent stimuli from the periodontal ligaments and the TMJ, enabling a response that affects the total motor output of the mandibular postural muscles. Head posture is also adjusted to facilitate a mandibular trajectory free from premature occlusal contacts as the mandible moves to $\mathrm{CO} .^{29}$ Although the CNS elaborates a habitual rest position that will reduce functional demands, it remains an accommodative response. ${ }^{24,25}$

After obtaining detailed information about habitual function, the next step is to determine the mandibular posture and path of closure without occlusal conditioning. To do this, the periodontal afferent inputs to the CNS are interrupted, allowing relaxation of the mandibular postural muscles. A TENS device can be used to relax the musculature and increase blood circulation. ${ }^{30,31}$

Tooth contact must be avoided during this procedure. Occlusal wax has been used for a number of years, although any occlusal interference media can be helpful as long as it causes a minimal degree of conditioning. Indeed, there will always be a certain level of conditioning and consequent accommodation as it is not possible to eliminate all afferent inputs to the CNS. An Aqualizer (Jumar Corp., Prescott, AZ, USA), which is a temporary diagnostic splint, is useful for producing a rapid relaxation of the masseter and temporalis muscles, and was used on the patient discussed in this case report. ${ }^{24,25}$
The TENS application lasts at least 45 minutes when the muscles are relaxed; as assessed using surface electromyography. The TENS device is then switched off, and the muscles are assessed using surface electromyography to ensure that the output is below normal limits. After the muscles have been relaxed, the Aqualizer can be removed, and the patient should be instructed not to close his or her teeth (to avoid occlusal reconditioning) and to remain relaxed. The TENS device is then switched on again, and the electrical output is increased. This produces a mandibular movement from an unconditioned rest position to closure (via an ideal path of closure or neuromuscular trajectory). The patient should then be instructed to close to $\mathrm{CO}$ so that the differences between the ideal neuromuscular function and the habitual conditioned movements can be evaluated.

Mandibular range and motion were recorded using the K7 Evaluation System (Myotronics-Noromed, Inc., Kent, WA, USA; Fig. 1A). This device includes the K7/Computerized Mandibular Scanner (CMS), the K7/Electromyograph (EMG), and the $\mathrm{K} 7 /$ Electrosonograph (ESG; for joint sound recording), and is regularly checked and calibrated at two-year intervals. The device allows mandibular movements and function to be evaluated simultaneously (Fig. 1A). The software allows the clinician to evaluate electromyographically selected muscles during mandibular movements, such as clenching or mouth opening.

The J4 Myomonitor TENS unit (Myotronics-Noromed, Inc.) was used in the study. The J4 is a low-frequency TENS device that delivers bilateral electrical stimulation of the trigeminal (V) and facial (VII) cranial nerves when electrodes are placed bilaterally on the coronoid notch. A third electrode, the neutral electrode, is placed at equal distance from the first two, behind the lower neck (Fig. 1B).

The aim of this paper is to provide an illustration of neuromuscular orthodontics as a diagnostic and therapeutic procedure that enables skilled practitioners to understand the physiology of their patients in more depth and to select treatment procedures according to specific neuromuscular orthodontic guidelines.

Figure 1A, 1B. Photographs of $(A)$ the system for mandibular tracking and electromyography and (B) the TENS device. TENS: transcutaneous electrical nerve stimulation

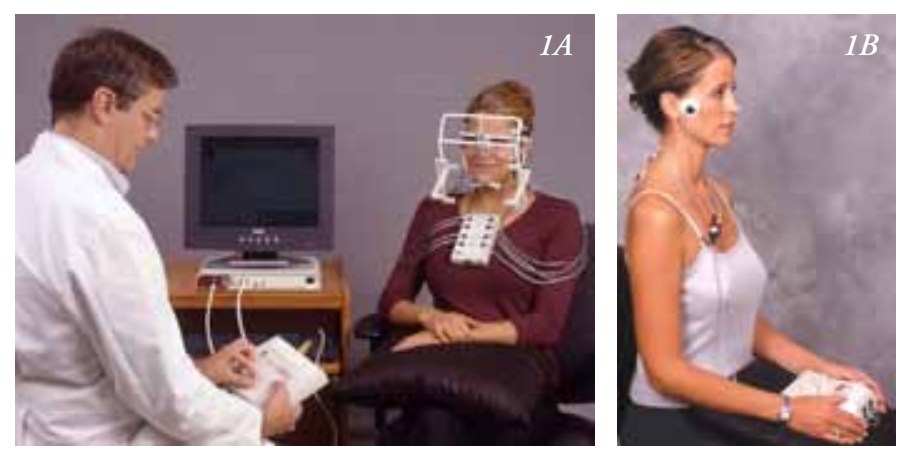




\section{CASE REPORT}

\section{Case description}

\section{Diagnosis}

An 11-year-old Caucasian boy sought orthodontic treatment at the office for a recurrent pain of the right TMJ, misaligned teeth, and irregular clicking noises (associated with the right TMJ) during mouth opening. He had a concave profile and thin lips (Fig. 2A). The patient reported that he was allergic to nickel. Onychophagy (nail biting) was the only parafunction present.

TMJ joint evaluation revealed a high-frequency clicking sound on the right-hand side during normal habitual full aperture of the mouth. No noise was observed on the left TMJ. Tenderness was observed during palpation of the right TMJ and anterior right temporal region.

The patient reported that the pain in the right TMJ had started approximately 6 months prior to the consultation, and was mostly continuous and dull with occasional acute, intense intervals. The patient reported increased acute pain during chewing. Antiinflammatory drugs did not resolve the symptomology and only provided a slight transitory improvement.

Pre-treatment panoramic X-ray and lateral cephalograms were performed (Fig. 2C-6D). No observations relevant to the TMJ were made on either the panoramic or the lateral cephalogram. No other radiologic evaluation was considered necessary for TMJ evaluation.

Intraoral examination revealed the patient to have good oral hygiene and healthy periodontal tissues. The lower incisors were inclined lingually, and a deep bite was present. A Class I malocclusion was present on both sides (Fig. 2B-D).

Figure 2A, 2B. (A) Extra-oral photographs showing the patient's concave profile and thin lips and $(B)$ intra-oral photographs showing a Class I relationship on both sides.
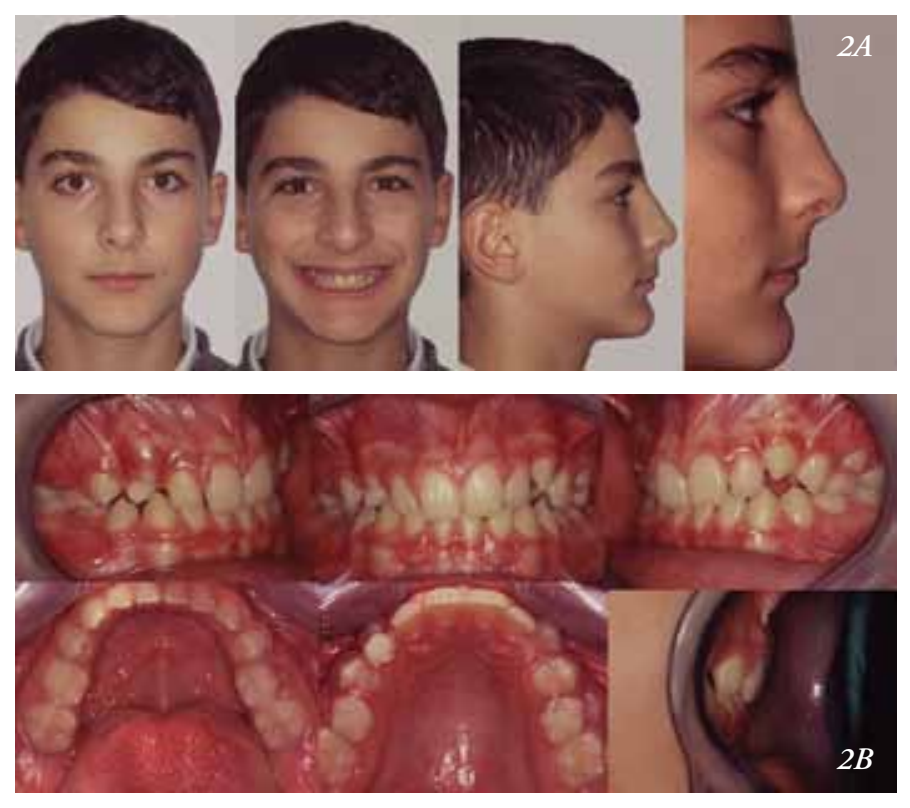

Figure 2C. Panoramic X-ray before treatment.

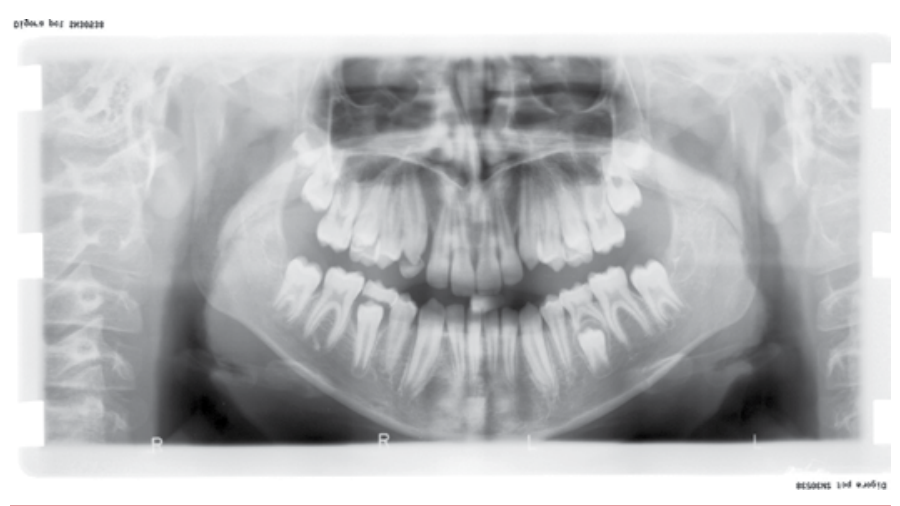

Figure 2D.

Lateral cephalogram

before treatment.

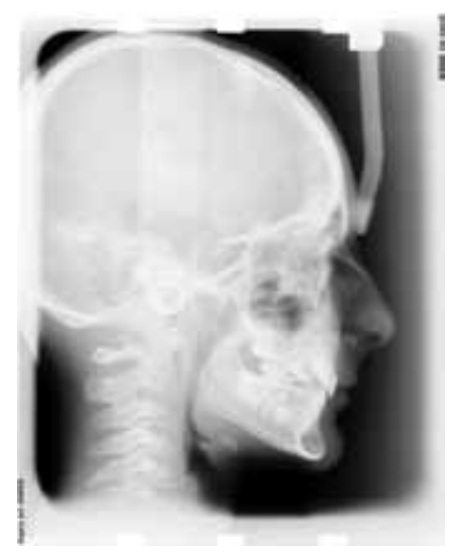

Figure 2E. Panoramic X-ray after treatment.

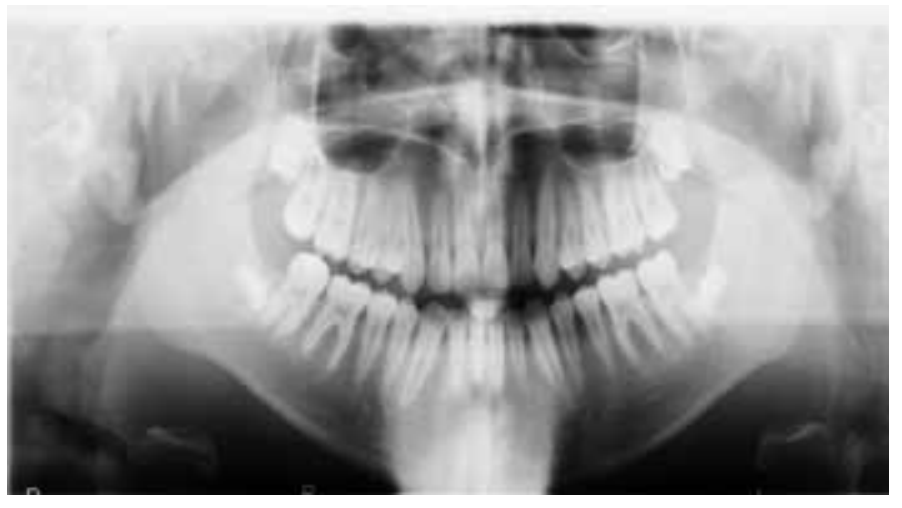

Figure 2F.

Lateral cephalogram

after treatment.

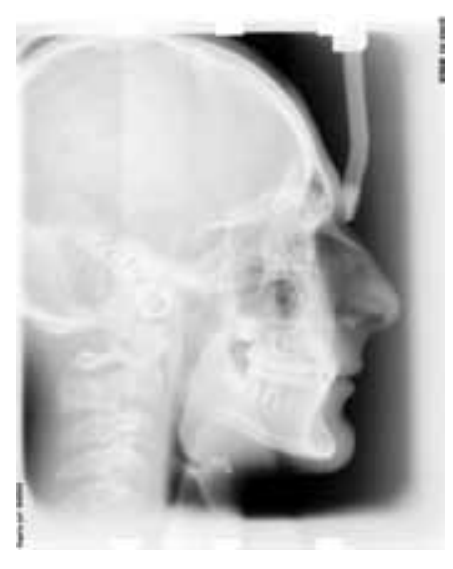


Figure 3A. Scan 1 (pre-treatment) showing that the degree of mouth opening was satisfactory (the total mouth aperture was approximately $40 \mathrm{~mm}$ ), there was a slight lateral deviation to the left upon opening $(1.8 \mathrm{~mm})$, and the velocity at tooth contact was also satisfactory. AP: anterior-posterior; Vert: vertical.

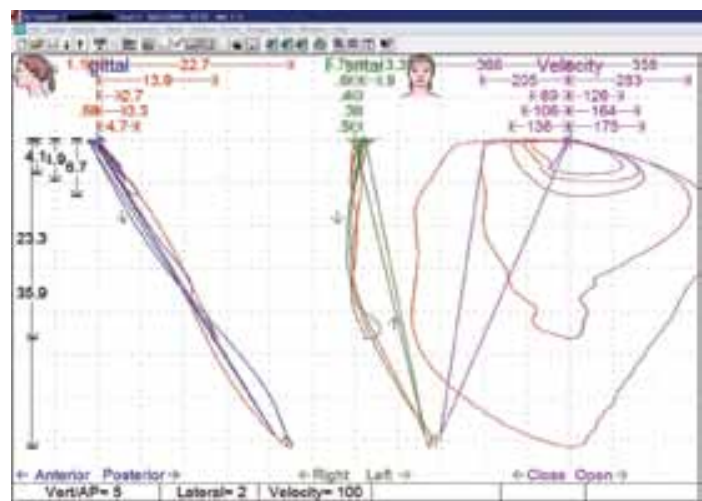

Figure 3B. Scan 3 (pre-treatment) showing the vertical, anterior-posterior, and lateral movements of the mandible in sweep mode. The patient was instructed to maintain the mandible in the rest position and then to close it to $C O$. There was a slight excess in freeway space $(2.9 \mathrm{~mm})$. When closing, there was no apparent anterior-posterior movement, a slight movement forward upon closure (which was immediately converted to a backward movement [black arrow]), and a lateral movement that was initially to the left of the horizontal reference plane. AP: anterior-posterior; Lat: lateral; Ver: vertical.

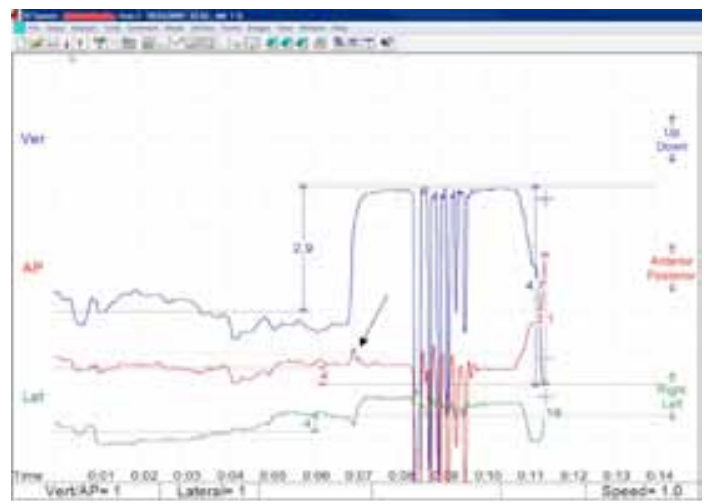

Figure 3C. Scan 9 (pre-treatment) showing the surface EMG results for the LTA, $R T A, L M M$, and RMM. The muscle output at rest was within the normal limits. CO: centric occlusion; EMG: electromyography; LMM: left masseter muscle; LTA: left anterior temporalis; RMM: right masseter muscle; RTA: right anterior temporalis.

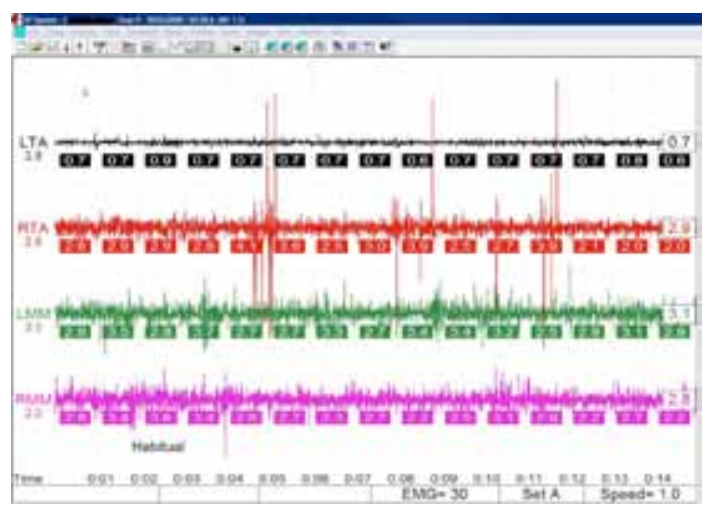

Figure 3D. Scan 11 (pre-treatment) showing the surface EMG results in sweep mode. The patient was instructed to swallow (first burst), clench in CO (second burst), and then clench with cotton rolls between the teeth, which temporarily prevented occlusion (third burst). Note that during swallowing, the temporalis muscles were slightly unbalanced. During clenching in $C O$, there was cross-pattern firing. The use of cotton rolls reduced the muscle balance discrepancy. CO: centric occlusion; EMG: electromyography; LMM: left masseter muscle; LTA: left anterior temporalis; $R M M$ : right masseter muscle; $R T A$ : right anterior temporalis.

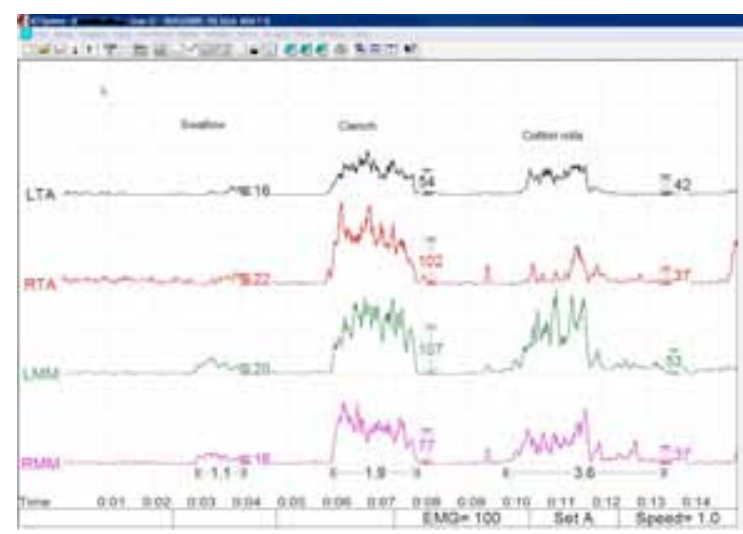

Figure 3E. Scan 21 (pre-treatment) showing the vertical, anterior-posterior, and lateral movements of the mandible in sweep mode during filtered EMG and swallowing. The patient was instructed to swallow normally and then close to CO, making a "tap-tap" motion. During swallowing, the patient did not move to $C O$, indicating that the swallowing occurred with the tongue between the teeth. The RTA was associated with higher output than the LTA during swallowing. AP: anterior-posterior; DEG: deglutition; CO: centric occlusion; EMG: electromyography; Lat: lateral; LMM: left masseter muscle; LTA: left anterior temporalis; RMM: right masseter muscle; RTA: right anterior temporalis; Vert: vertical.

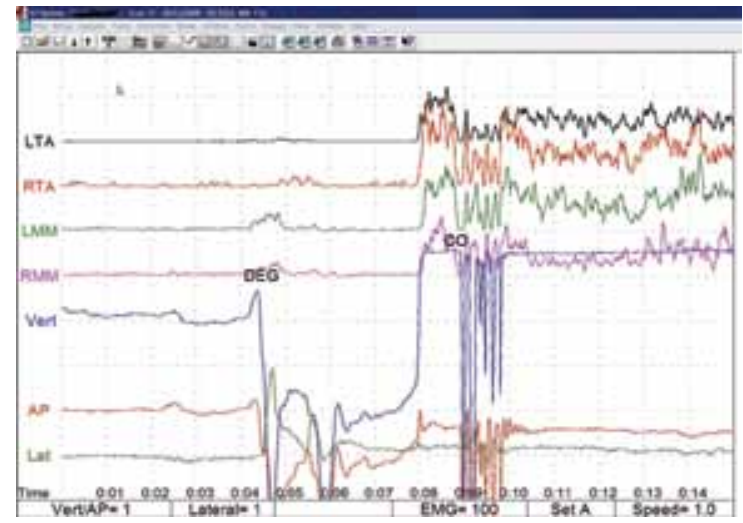


Figure 3F. Scan 13 (pre-treatment) showing the range of motion, opening posteriorly and closing anteriorly. AP: anterior-posterior; Vert: vertical.

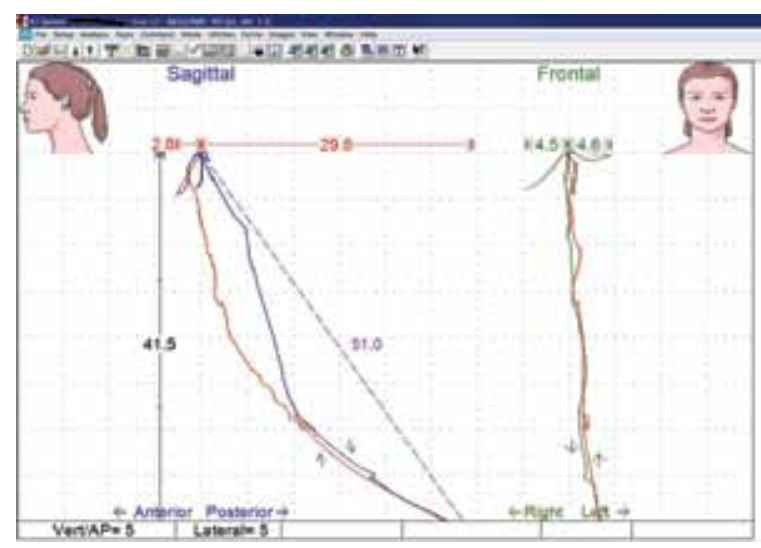

Figure 3G. Scan 9 (pre-treatment) showing that the muscles were relaxed (after TENS). An Aqualizer was used to avoid tooth contact for CNS deconditioning. CNS: central nervous system; LMM: left masseter muscle; LTA: left anterior temporalis; RMM: right masseter muscle; RTA: right anterior temporalis; TENS: transcutaneous electrical nerve stimulation.

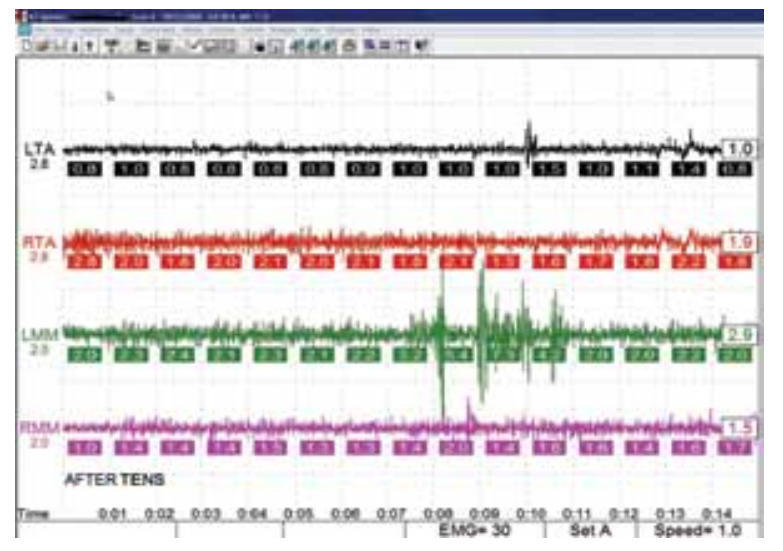

Figure 3H. Scan 415 (pre-treatment) showing that, as the mandible moved from the rest position to $C O$, the computer calculated that $1.3 \mathrm{~mm}$ of overjet was needed on the horizontal plane in order to resolve the mandibular retrusion. The black dot on the sagittal view represents the ideal occlusion, with $1.8 \mathrm{~mm}$ of freeway space (the myocentric position). AP: anteriorposterior; CO: centric occlusion; Vert: vertical.

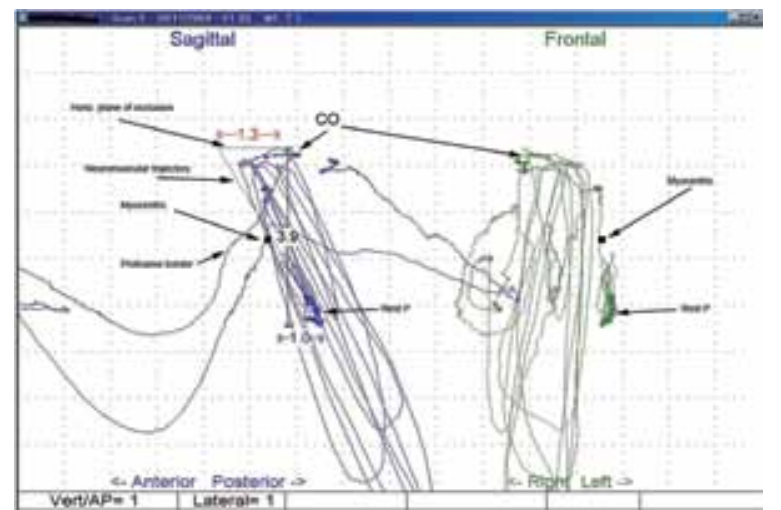

Figure 3I. Scan 4/5 (pre-treatment) showing a partial view of scan 5 in split-screen mode. On the left-hand side, the patient was instructed to close from resting position to $C O$. The AP movement was forward (up) because of muscle deconditioning. On the right, the same tracing is shown from the sagittal and frontal views and shows a premature contact with upper lateral left incisor. AP: anterior-posterior; CO: centric occlusion; Lat: lateral; Spd: speed; Vert: vertical.

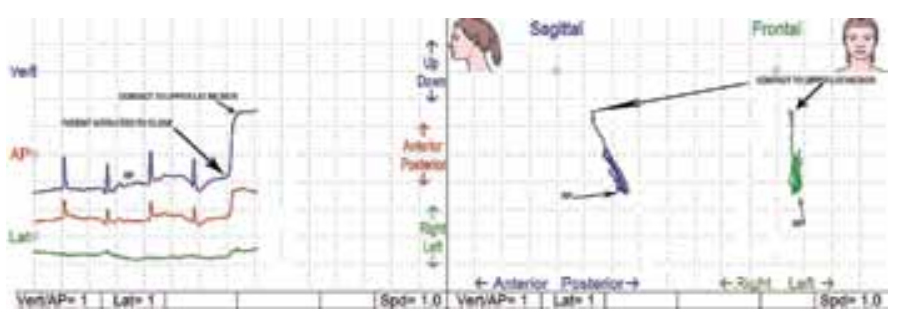

Figure 3J. Scan 5 (pre-treatment) showing that, after initial tooth contact, the mandible slid backward and right to CO. AP: anterior-posterior; CO: centric occlusion; Lat: lateral; Spd: speed; Vert: vertical.

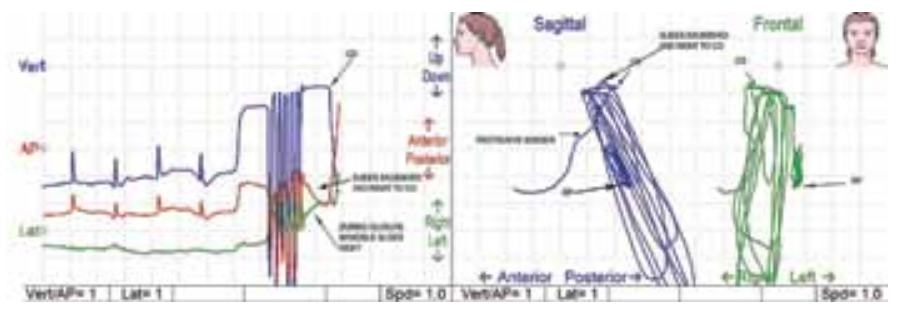

Figure 3K. Scan 4 (pre-treatment) showing a sweeping view of scan 5 and providing a complementary analysis. After relaxation/deconditioning, the mandible came forward from the rest position and did not avoid tooth contact. As the patient was instructed to "tap" to CO, the mandible closed upward to $C O$ while sliding back. From the rest position to $C O$, the mandible closed approximately $2 \mathrm{~mm}$ to the right. AP: anterior-posterior; CO: centric occlusion; Lat: lateral; Vert: vertical.

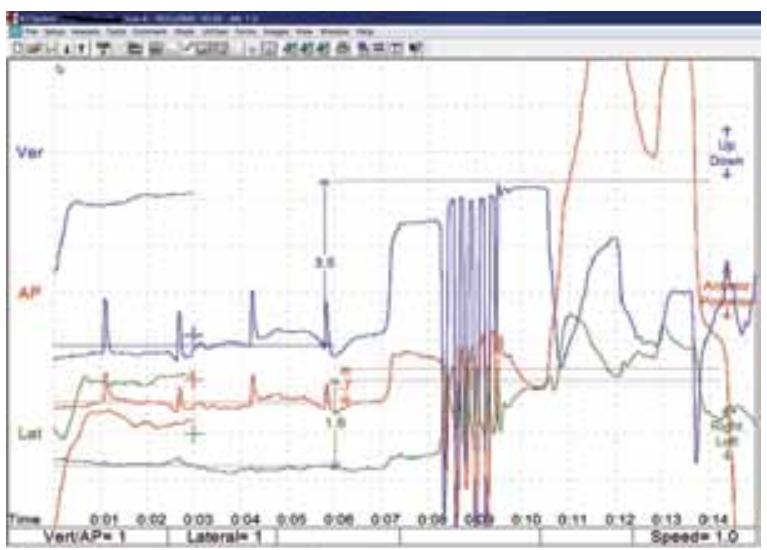


Figure 4. Intra-oral photographs showing a canine Class II relationship.

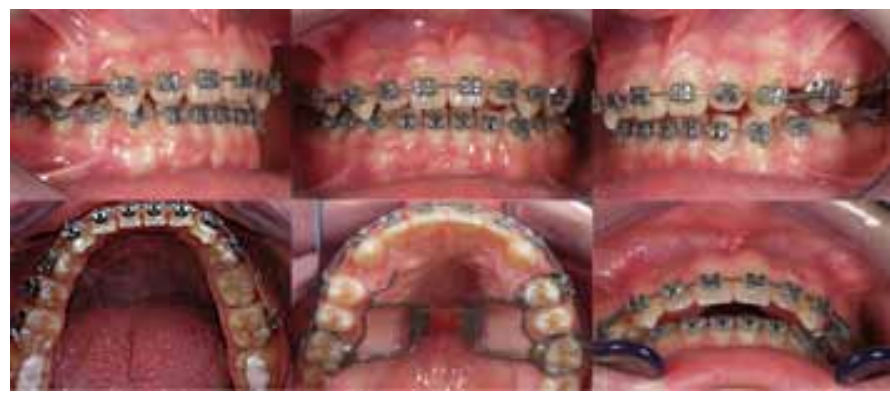

Figure 5. Intra-and extra-oral photographs at the end of treatment.

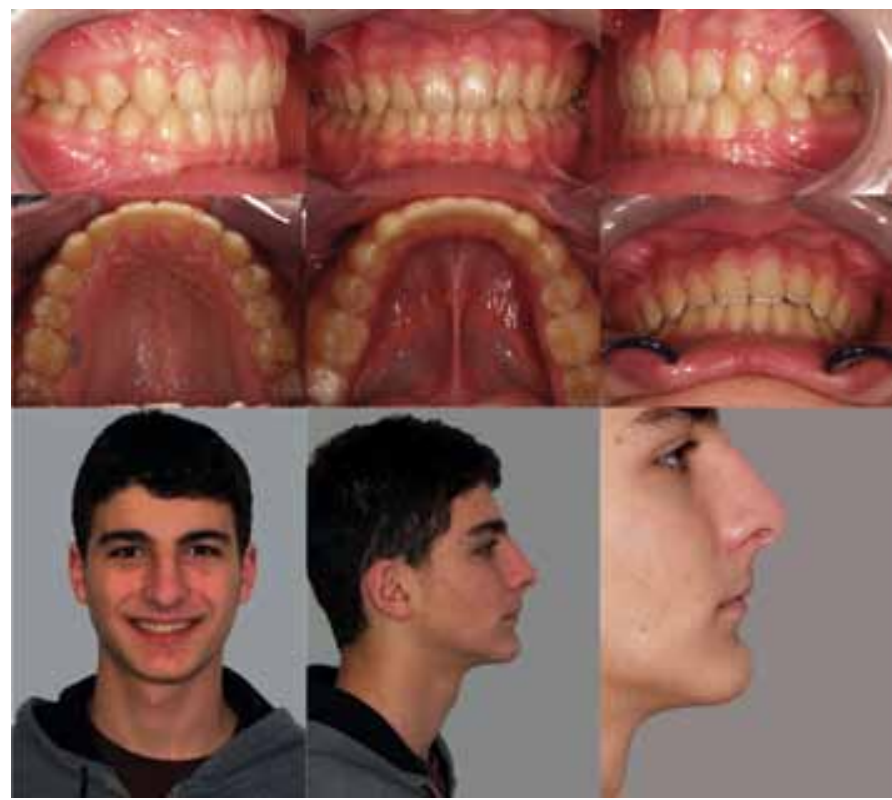

Figure 6A. Scan 1 (post-treatment) showing that the sagittal, frontal, and velocity tracings were within the normal limits. The speed at tooth contact to $C O$ was very good. AP: anterior-posterior; CO: centric occlusion; Vert: vertical.

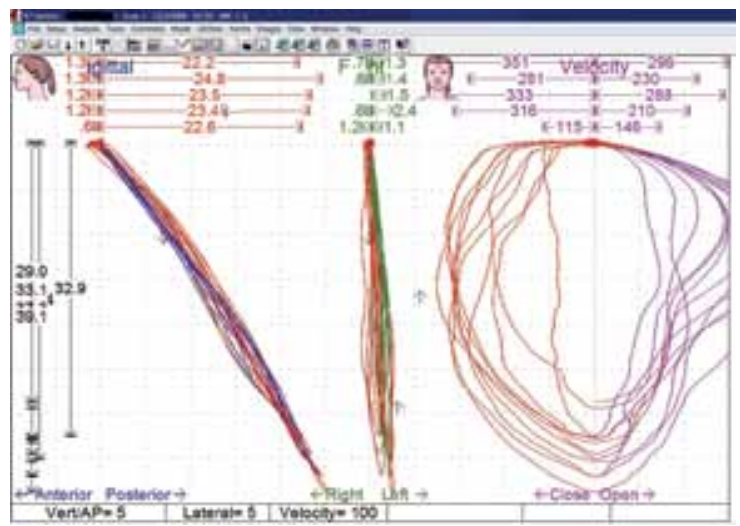

Figure 6B. Scan 3 (post-treatment) showing that the freeway space was 3 $\mathrm{mm}$, the mandible moved forward during closure (A/V ratio: 0.4$)$, and the lateral movement was $0.1 \mathrm{~mm}$ to the right. AP: anterior-posterior; $A / V$ : anterior/vertical ratio; Lat: lateral; Vert: vertical.

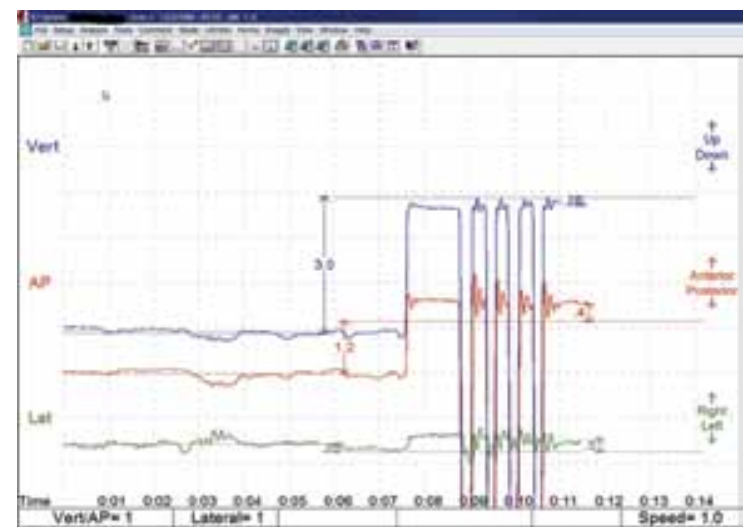

Figure 6C. Scan 11 (post-treatment) showing that muscle function was improved (with balanced masseters and unbalanced temporalis muscles) and the overall output was improved. EMG: electromyography; LMM: left masseter muscle; LTA: left anterior temporalis; RMM: right masseter muscle; RTA: right anterior temporalis.

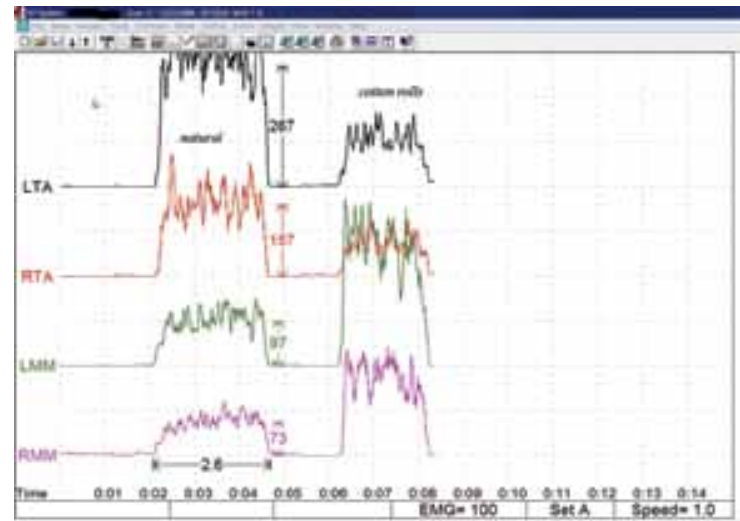

Figure 6D. Scan 9 (post-treatment) showing the muscle activity before relaxation with the Aqualizer and TENS. All the values were within the normal limits. EMG: electromyography; LMM: left masseter muscle; LTA: left anterior temporalis; RMM: right masseter muscle; RTA: right anterior temporalis; TENS: transcutaneous electrical nerve stimulation.

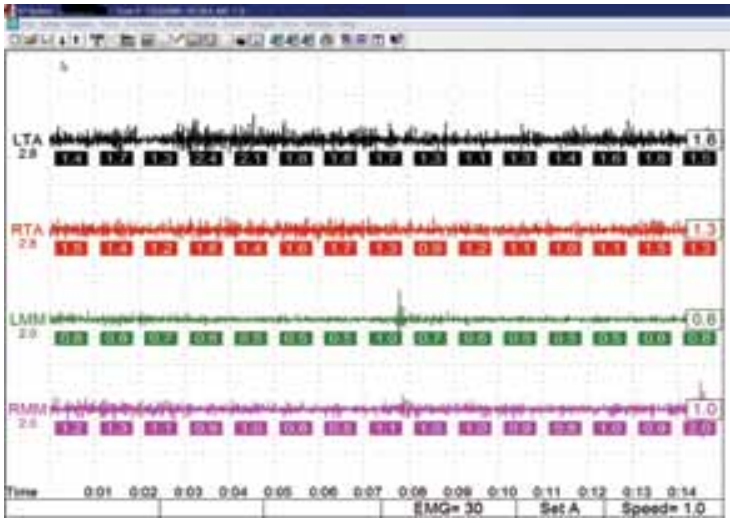




\section{Treatment objectives}

The treatment objectives were to resolve the TMJ pain and establish a healthy occlusion in the patient. Since TMJ pain was the major complaint, the patient underwent a complete functional analysis, which enabled the calculation of the degree of mandibular advancement needed to correct the TMJ dysfunction. The first step was to achieve mandibular freedom: that is, to avoid any occlusal interference that limits the spontaneous anterior repositioning of the mandible. Initially, expansion and bonding only involved the upper arch. Expansion and alignment of the upper incisors and canines is the first step towards spontaneous mandibular advancement. The patient was made aware that the duration of the treatment would be longer than usual due to the use of nickel-free orthodontic appliances.

\section{Treatment alternatives}

The treatment alternatives included: no treatment; orthodontic treatment; or the application of a neuromuscular orthotic. The patient refused treatment that was based on the use of a mandibular repositioning device and preferred orthodontic treatment.

\section{Functional analysis}

Pre-treatment functional analysis was carried out in November 2004 during a single site visit. The results of this functional analysis are shown in Fig. 3A-3K.

Figure $3 \mathrm{~A}$ shows that the degree of mouth opening was satisfactory (the total mouth aperture was approximately 40 $\mathrm{mm}$ ), that there was a slight lateral deviation to the left upon opening $(1.8 \mathrm{~mm})$, and that the velocity at tooth contact was also satisfactory. Figure $3 \mathrm{~B}$ shows that the anterior component of the mandibular trajectory during closure from the habitual rest position to $\mathrm{CO}$ is undetectable. In a normal path of closure from any degree of opening, the mandible will always close forward and anteriorly, as the condyle rotates in the fossa. The resulting rest position is the answer that the CNS has given to the neuromuscular requests. This abnormal vertical path to $\mathrm{CO}$ from habitual rest position is an adaptive response of the CNS to avoid any premature contact with the upper incisors. In fact, the adaptive responses of muscle function must result in minimizing premature tooth contacts ${ }^{25}$, and this muscle programming involves a complex activation and coordination organized by the CNS to remember how to avoid noxious premature contacts.

Figure 3D shows cross-pattern firing and unbalanced muscle function during clenching. After deconditioning, Figures
$3 \mathrm{H}-\mathrm{J}$ (scans 4 and 5) illustrate how the neuromuscular system would act during an ideal neuromuscular function. During the closure, the mandible came forward to a more forward position with respect to the horizontal plane of occlusion. After deconditioning, the instruction to the patient to close to $\mathrm{CO}$ ("close your teeth so that the back teeth touch") resulted in premature tooth contact of the front incisors before reaching $\mathrm{CO}$. This is due to the fact that the neuromuscular system has temporarily lost the muscle memory, and the patient cannot close easily to $\mathrm{CO}$ whilst avoiding premature contacts.

One of the main objectives of the orthodontic treatment was not only to achieve satisfactory aesthetic results but also to create a functional occlusion and preserve TMJ function. Since the diagnostic procedure established that the mandible was distally occluded (Fig. $3 \mathrm{H}$ ), this had to be taken into consideration when calculating the space needed for lower incisor alignment. It was determined that, without a major change in freeway space, at least $1.3 \mathrm{~mm}$ of overjet would have to be added to the overjet required for lower alignment. It was established that a minimum of $2 \mathrm{~mm}$ of overjet was required for lower incisor correction, and therefore, a total of approximately $3.3 \mathrm{~mm}$ of overjet was needed before lower alignment (Fig. 2B).

There was likely to be some residual growth in the patient, which was taken into consideration. However, even achieving a 4.5-mm overjet (Fig. 4) was insufficient to achieve an ideal neuromuscular occlusion at the end of treatment (Fig. 6F- 6H) and 6 years after retention (Fig. 8G). The ideal neuromuscular request should have been a more forward positioning of $\mathrm{CO}$ on the horizontal plane of occlusion, respectively $0.8 \mathrm{~mm}$ (Fig. $6 \mathrm{~F}-6 \mathrm{H}$ ) and $1.5 \mathrm{~mm}$. (Fig. 8G). Although the neuromuscular objectives would ideally include an overlapping of pre- and postTENS scans at the end of treatment and follow-up, the increase in muscle recruitment during clenching, and the reduction of asymmetry as confirmed by surface electromyography before and after treatment, confirm success in orthodontic treatment $(3 \mathrm{D}, 6 \mathrm{C}, 8 \mathrm{E},) . .^{32-34}$

\section{Treatment progress and outcomes}

The treatment was started in December 2004 with an upper palatal expander. The expansion cycle involved two screw activations per day. In April 2005, the upper arch was bonded with equilibrium brackets (Dentaurum GmbH \& Co. KG, Ispringen, Germany) and Noninium 0.014" wire (Dentaurum GmbH \& Co. KG). Subsequently, 0.016-0.018" and 0.017" $\times 0.025^{\prime \prime}$ titanium-molybdenum alloy archwires were selected. Coil springs were added to the upper arch in October 2005, while upper anchorage was guaranteed by the upper palatal expander. The lower arch was bonded in March 2006 (only 
after there was sufficient overjet to avoid any occlusal interference with the upper arch) (Fig. 4). Noninium 0.018$0.016^{\prime \prime} \times 0.022^{\prime \prime}$ archwires were selected for the lower arch. Note that the canines had a Class II dental relationship.

Active treatment was finished in December 2007, and retention involved a lower fixed composite and upper removable appliance (Fig. 5). The patient had been asymptomatic since November 2005, and no clicking noises were present from this point onward. A post-retention functional analysis, the results of which are shown in Figure 6A-6H, was performed in February 2008 to assess whether the treatment objectives had been met.

In June 2015, the patient returned for a follow-up appointment. At this point, the patient had been asymptomatic since November 2005, and he had been out of retention for 6 years. Intra- and extra-oral photographs were taken, as shown in Figure 7, and a second post-retention functional analysis was conducted, the results of which are shown in Figures 8A-8G. Occlusal stability, muscle function, and TMJ function had been preserved, and there were no changes in parafunctional habits. These results are in accordance with previous studies that confirm the efficacy of the neuromuscular approach. ${ }^{1,35-37}$

\section{DISCUSSION}

Neuromuscular approaches have the potential to benefit large numbers of patients in the oral setting, although they remain seldom employed, perhaps due to the equipment, expertise and costs associated with the procedures. That said, neuromuscular concepts have been used to treat temporomandibular disorder for more than 30 years in isolated settings. In one case from Japan, 6and 23-year follow-up data illustrated a patient benefit of having an occlusion corrected using a minimally-invasive and reversible approach based on electronic stimulation, monitoring and neuromuscular concepts. ${ }^{38}$ Other studies have reported similar results, and illustrated how myocentric relaxation - using TENS and holistic techniques - have been used to help the TMJ bear the burden of occlusions at neuromuscular rest. ${ }^{39}$ Neuromuscular orthodontics specifically is further behind than dentistry as a whole, with only a limited number of studies have published results obtained by such approaches, although reports of pain alleviation and dysfunction correction breed encouragement. ${ }^{36}$ Here, we have also shown the utility of such approaches to deal with complex clinical problems for patient benefit. Together, our studies provide to a growing body of evidence that $\mathrm{NO}$ techniques and practices merit further clinical research exploration and application to treat a range of orthodontic and conformational problems associated with the oral region. Importantly, wider use of such approaches will advance the technology, lower costs and improve treatment.
Figure 6E. Scan 10 (post-treatment) showing the muscle activity at rest after 45 minutes of relaxation with TENS. All the values were within the normal limits. EMG: electromyography; LMM: left masseter muscle; LTA: left anterior temporalis; RMM: right masseter muscle; RTA: right anterior temporalis; TENS: transcutaneous electrical nerve stimulation.

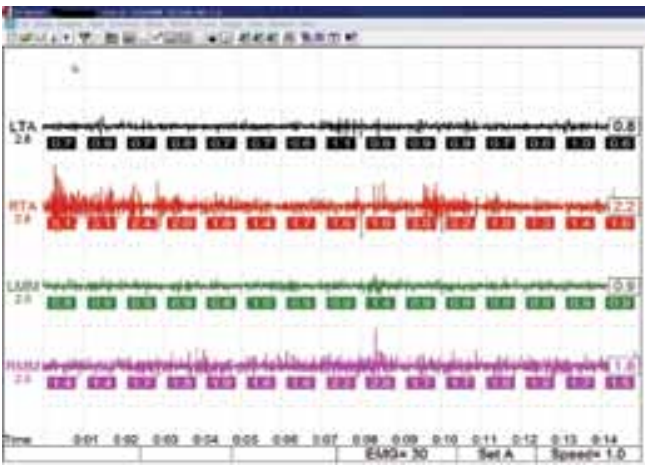

Figure 6F. Scans 4 and 5 (post-treatment) showing that the ideal path to closure was just $0.8 \mathrm{~mm}$ anterior of the $\mathrm{CO}$ on the horizontal plane. The protrusive border was steep. The red AP line in sweep mode shows there was no retrusion of the mandible during the closure. AP: anterior-posterior; CO: centric occlusion.

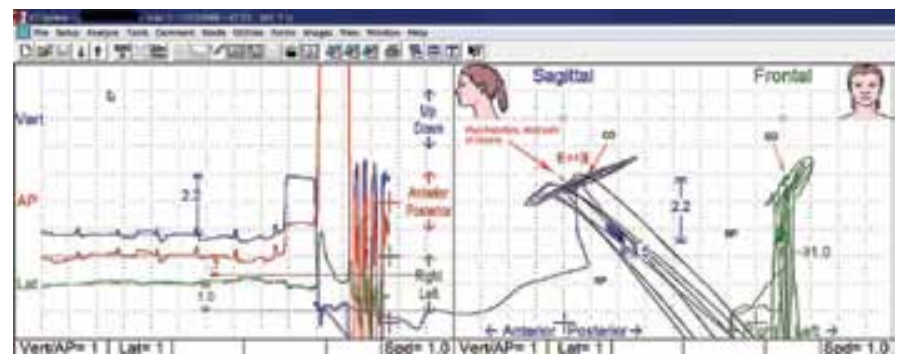

Figure 6G. Partial view of scans 4 and 5 (post-treatment) clarifying the slight posterior slide of the mandible at tooth contact. AP: anterior-posterior; Lat: lateral; Spd: speed; Vert: vertical.

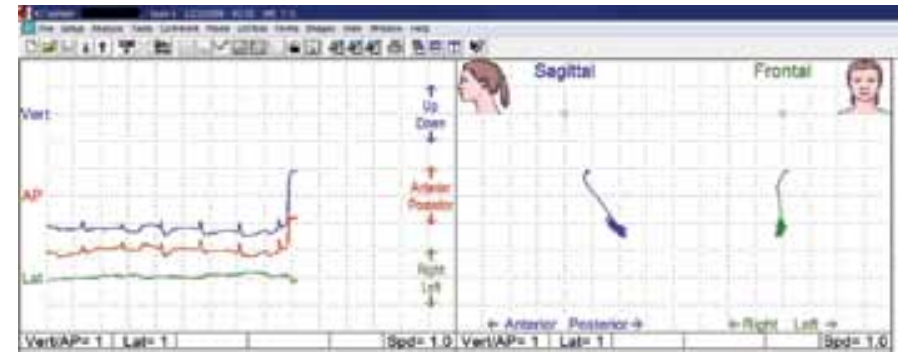

Figure 6H. Partial view of scans 4 and 5 (post-treatment) clarifying the steepness of the anterior incisors. AP: anterior-posterior; Lat: lateral; Spd: speed; Vert: vertical.

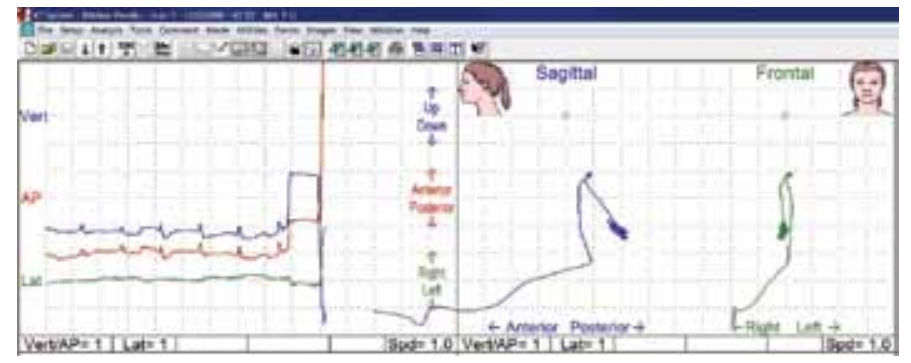


Figure 7. Intra-and extra-oral photographs at 7.5 years after treatment.

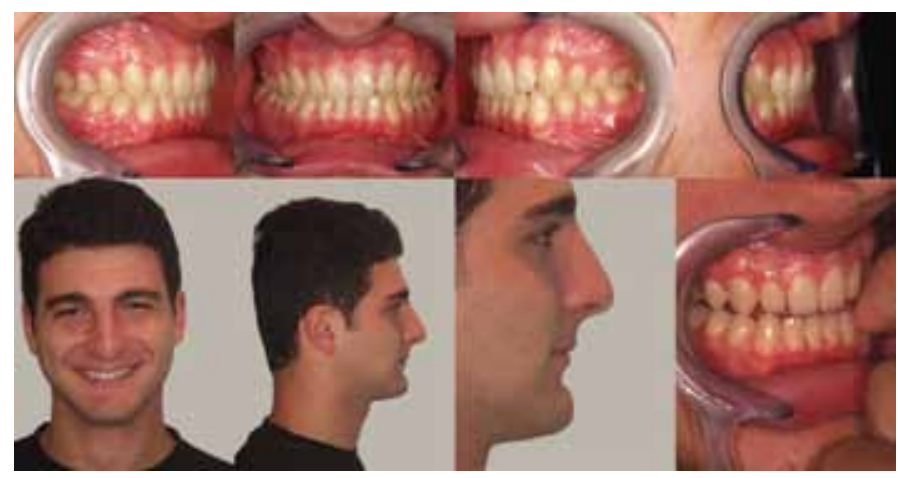

Figure 8A. Scan 1 (post-treatment follow-up) showing that all the values were within the normal limits, the speed at tooth contact was high, and the movement was fluent. AP: anterior-posterior; Vert: vertical.

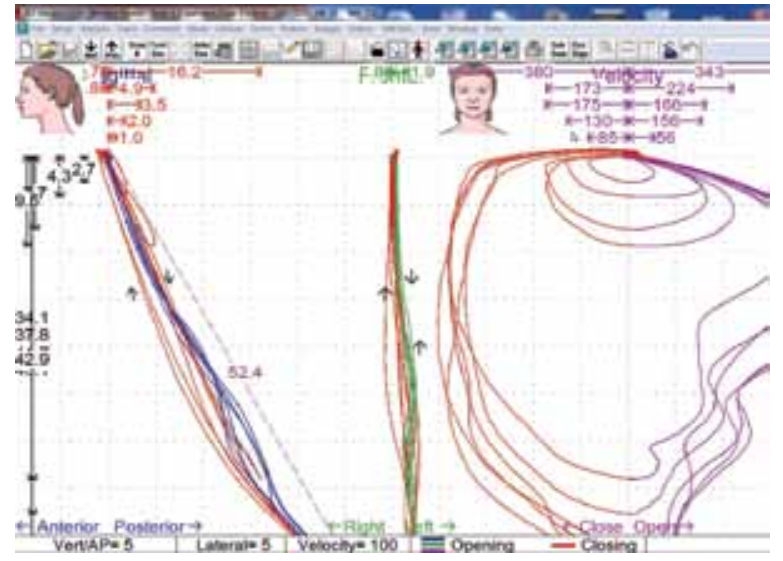

Figure 8B. Scan 3 (post-treatment follow-up) showing that the freeway space was $2.8 \mathrm{~mm}$, the mandible came forward $1.6 \mathrm{~mm}$ ( $A / V$ ratio: 0.5$)$, and there were no significant lateral movements. AP: anterior-posterior; $A / V$ : anterior/vertical ratio; Lat: lateral; Vert: vertical.

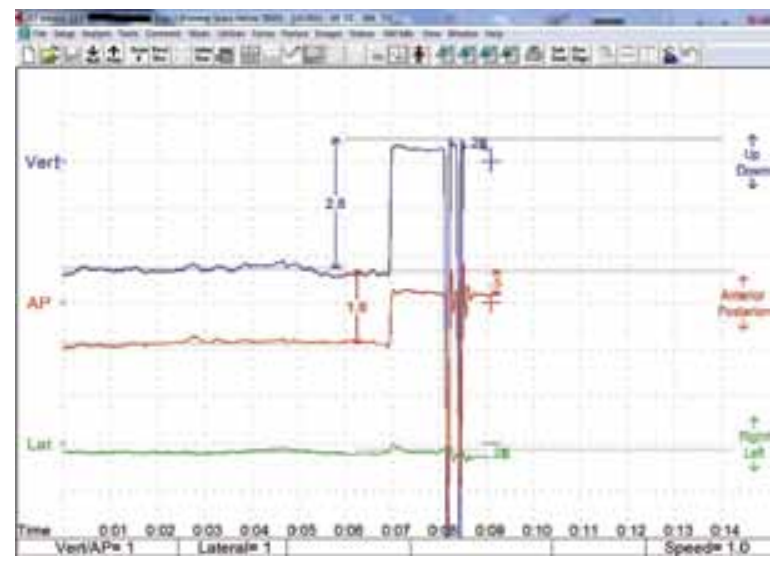

Figure 8C. Scan 21 (post-treatment follow-up) showing an improvement in the patient's swallow pattern. AP: anterior-posterior; EMG: electromyography; Lat: lateral; LMM: left masseter muscle; LTA: left anterior temporalis; RMM: right masseter muscle; RTA: right anterior temporalis; Vert: vertical.

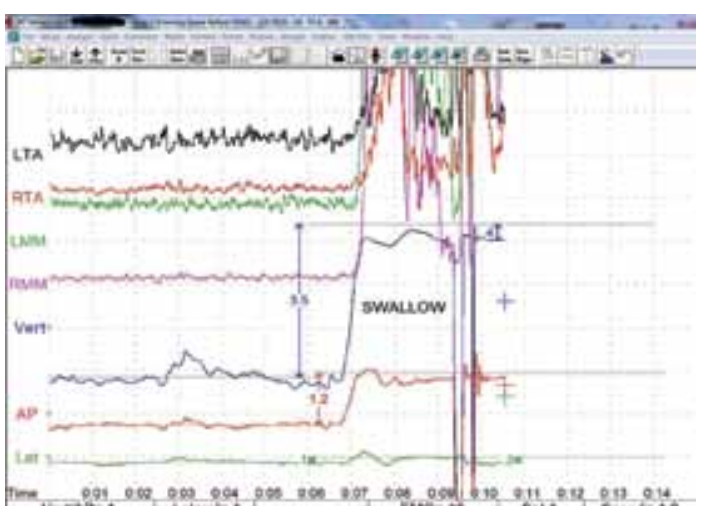

Figure 8D. Scan 9 (post-treatment follow-up) showing the EMG results before deconditioning with TENS. The LMM and LTA results at rest showed output that was slightly above the normal limits. EMG: electromyography; LMM: left masseter muscle; LTA: left anterior temporalis; RMM: right masseter muscle; RTA: right anterior temporalis; TENS: transcutaneous electrical nerve stimulation.

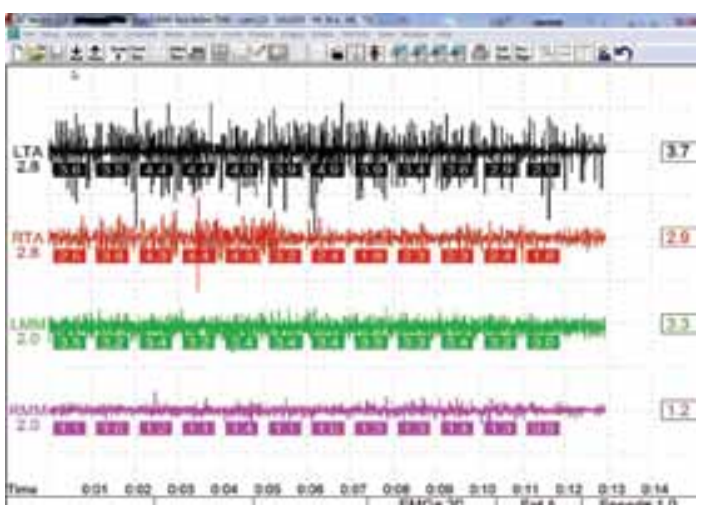

Figure 8E. Scan 11 (post-treatment follow-up) showing natural clenching (ideal balanced function during clenching, with both masseters and temporalis muscles well balanced) and cotton-roll clenching (no change in the general output pattern). The output of the masseters was higher than that of the temporalis muscles. EMG: electromyography; LMM: left masseter muscle; LTA: left anterior temporalis; RMM: right masseter muscle; $R T A$ : right anterior temporalis.

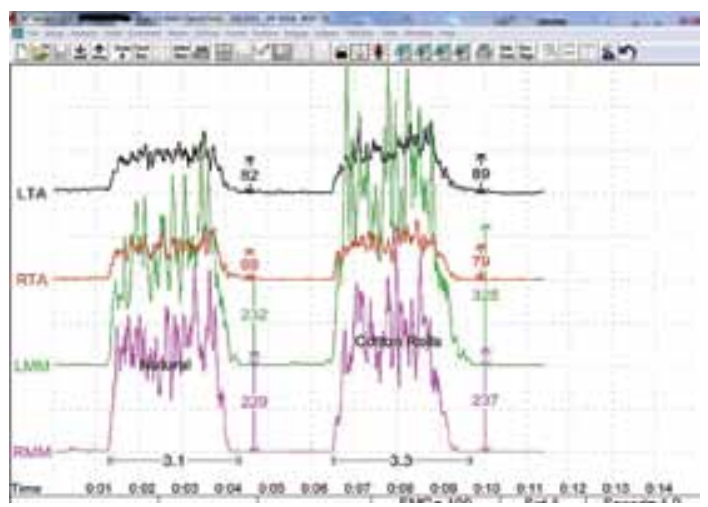


Figure 8F. Scan 10 (post-treatment follow-up) showing the EMG after deconditioning with the Aqualizer and TENS. All the values were below the normal limits. EMG: electromyography; LMM: left masseter muscle; LTA: left anterior temporalis; RMM: right masseter muscle; RTA: right anterior temporalis; TENS: transcutaneous electrical nerve stimulation.

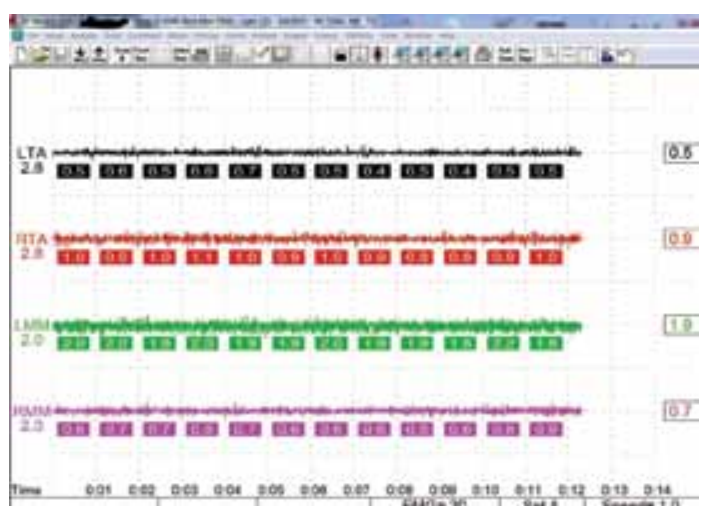

Figure 8G. Scans 4 and 5 (post-treatment follow-up) showing that although the physiological rest position was $0.6 \mathrm{~mm}$ posterior to the $\mathrm{CO}$, the neuromuscular trajectory was still projected with respect to the horizontal plane of occlusion by $1.5 \mathrm{~mm}$. The incisors were too steep; this slight relapse is most probably due to incisor lingual tipping. The mandible came forward by $0.5 \mathrm{~mm}$ during the closure. AP: anterior-posterior; A/V: anterior/vertical ratio; CO: centric occlusion; Lat: lateral; Spd: speed; Vert: vertical.

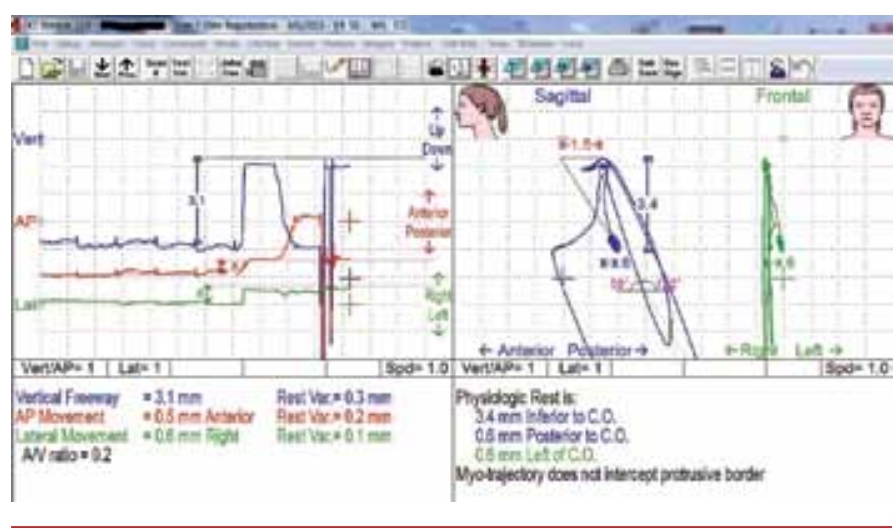

Figure 9. Jefferson-Sassouni cephalometric analysis before treatment.

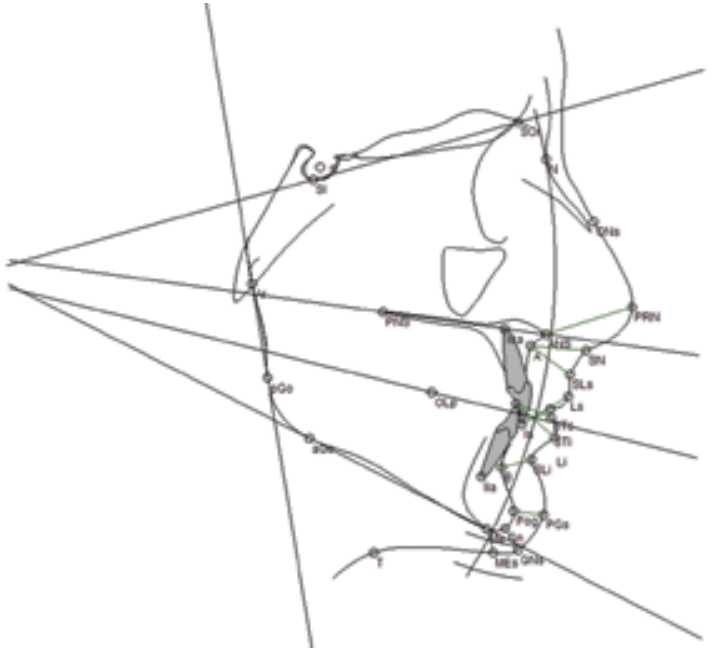

Figure 9.

Analysis-specific markers

SI: sella inferior

SOr: supraorbitale

Standard markers

S: sella turcica

$N$ : nasion

PNS: posterior nasal spine

ANS: anterior nasal spine

Is: incisor superior (upper incisor tip)

Ii: incisor inferior (lower incisor tip)

Pog: pogonion

Me: menton

aGo: anterior gonion (lower border of mandible)

$p$ Go: posterior gonion (posterior point on ramus)

Ar: articulare

OLp: occlusal line, posterior point

Lines:

$C P$ : cranial plane, a line defined by the markers $\langle S I\rangle$ and $\langle S O r\rangle$.

$R L$ : ramus line, a line defined by the markers $\langle A r\rangle$ and $\langle p G o\rangle$.

$A A r c$ : anterior arc, an arc with its center around $\langle O\rangle$, going through the marker

$<N>$, between the markers $\langle N>$ and $<M e>$.

VArc: vertical arc, an arc with its center around $\langle A N S>$, going through the marker $<$ SOr $>$, between the markers $<$ Pog $>$ and $<M e>$.

Varc-10: vertical arc $+10 \mathrm{~mm}$, an arc drawn as $<$ Varc $>$, with a 10-mm larger radius. The Jefferson analysis is a modified Sassouni analysis that consists of the following measurements:

ANS to AArc: the distance between the marker $\angle A N S>$ and the anterior arc. $0 \pm 3 \mathrm{~mm}$ Is to AArc: the distance between the marker $\langle I s>$ and the anterior arc. $0 \pm 3 \mathrm{~mm}$ Pog to AArc: the distance between the marker $\langle P o g\rangle$ and the anterior arc. $0 \pm 3 \mathrm{~mm}$ Lower facial height (LFH) diff: the distance between the marker $<M e>$ and the vertical arc. $0-10 \mathrm{~mm}$

ANS to AArc: $<O-A N S>$ minus $<$ AArc radius $>$. $0 \pm 3$

Is to AArc: $\langle O-I s>$ minus $<A$ Arc radius $>$. $0 \pm 3$

Pog to AArc: $<O-$ Pog $>$ minus $<$ AArc radius $>.0 \pm 3$

LFH diff: $<L F H>$ minus $<$ VArc radius $>$. 0-10

$A$ Arc radius: the distance between the markers $\langle O>$ and $\langle N\rangle$.

$O-A N S$ : the distance between the markers $\langle O\rangle$ and $\langle A N S\rangle$.

$O-I s$ : the distance between the markers $\langle O\rangle$ and $\langle I s\rangle$.

$O$-Pog: the distance between the markers $\langle O\rangle$ and $\langle$ Pog $\rangle$.

$V A r c$ radius: the distance between the markers $\langle A N S\rangle$ and $\langle S O r\rangle$.

LFH: the distance between the markers $\langle A N S\rangle$ and $\langle M e>$.

$C P$ : a line defined by the markers $\langle S I\rangle$ and $\langle S O r\rangle$.

$N L$ : a line defined by the markers $\langle P N S\rangle$ and $\langle A N S\rangle$.

$O L$ : a line defined by the markers $\langle O L p\rangle$ and $\langle O L a\rangle$.

Ola: a constructed marker at the midpoint between the markers $\langle I s\rangle$ and $\langle I i\rangle$.

$M L$ : a line defined by the markers $\langle a G o\rangle$ and $\langle M e>$.

$R L$ : a line defined by the markers $\langle A r\rangle$ and $\langle p G o\rangle$.

$c G$ : a constructed marker at the intersection between the lines $\langle R L>$ and $\langle M L\rangle$.

$O$ : a constructed marker at the intersection between the lines $\langle C P\rangle,\langle N L\rangle,\langle O L\rangle,\langle M L\rangle$.

AArc: an arc drawn around the marker $\langle O>$, through the marker $\langle N>$ and between the markers $\langle N\rangle$ and $\langle M e>$. Radius modification: 0.0

$V A r c$ : an arc drawn around the marker $<A N S>$, through the marker $<S O r>$ and between the markers $\langle$ Pog $\rangle$ and $\langle$ Me $\rangle$. Radius modification: 0.0

VArc-10: an arc drawn around the marker $\langle A N S\rangle$, through the marker $\langle$ SOr $\rangle$ and between the markers $\langle$ Pog $\rangle$ and $\langle$ Me $\rangle$. Radius modification: 10.0 
Figure 10. Jefferson-Sassouni cephalometric analysis after treatment.

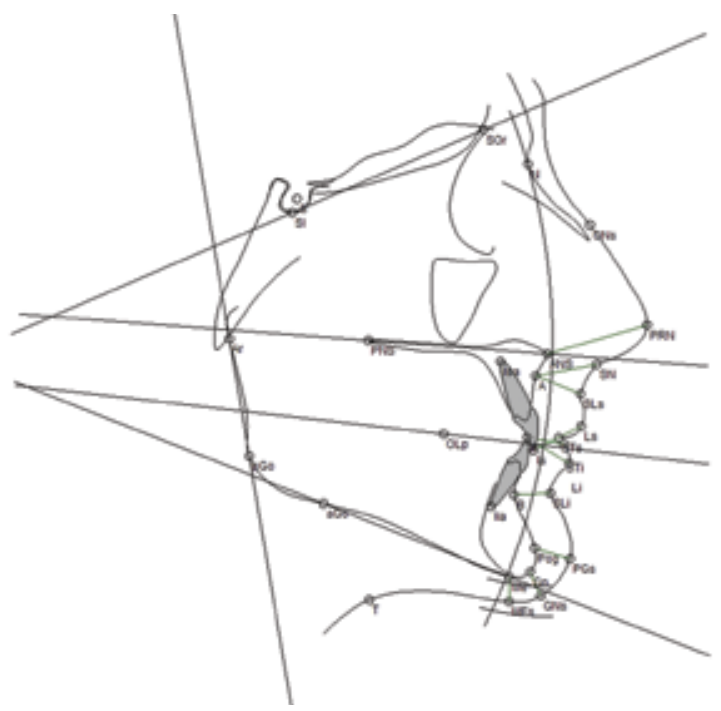

Figure 11. Superimposition on the cranial plane before and after treatment. Black: before treatment; red: after treatment.

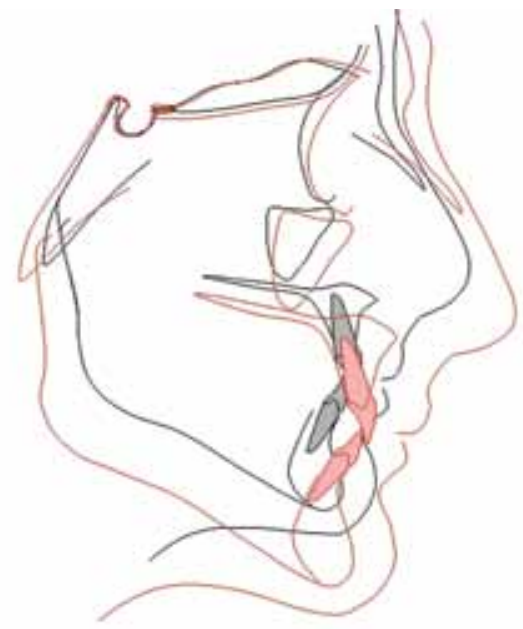

Figure 12. Jefferson-Sassouni cephalometric results. AArc: anterior arc; ANS: anterior nasal spine; Is: incisor superior; LFH: lower facial height; $O$ : a constructed marker at the intersection of the lines defined by a) the sella inferior and supraorbitale, b) the posterior and anterior nasal spines, c) the posterior point of the occlusal line and the midpoint between the upper and lower incisor tips, and d) the anterior gonion and menton; Pog: pogonion; sax 1: before treatment; sax2: after treatment; VArc: vertical arc.

\begin{tabular}{lrrll}
\hline Jefferson-Sassouni & & & \\
\hline Ceph name & $s a 4$ & $s a 2$ & Norm & Unit \\
\hline ANS to AArc & -1.9 & -1.9 & $0 \pm 3$ & $\mathrm{~mm}$ \\
\hline Is to AArc & -3.7 & $-3.20 \pm 3$ & $\mathrm{~mm}$ \\
Pog to AArc & 3.1 & $5.30 \pm 3$ & $\mathrm{~mm}$ \\
LFH diff & -3.3 & $-2.30-10$ & $\mathrm{~mm}$ \\
AArc radius & 183.6 & 211.8 & $\mathrm{~mm}$ \\
O-ANS & 181.6 & 209.9 & $\mathrm{~mm}$ \\
O-Is & 179.8 & 208.6 & $\mathrm{~mm}$ \\
O-Pog & 186.7 & 217.1 & $\mathrm{~mm}$ \\
VArc radius & 66.5 & 77.7 & $\mathrm{~mm}$ \\
LFH & 63.2 & 75.4 & $\mathrm{~mm}$ \\
& & & \\
\hline
\end{tabular}

\section{CONCLUSION}

Computerised mandibular scanning, electromyography, and TENS are important diagnostic aids that can facilitate diagnostic and treatment procedures in orthodontics. Here we illustrate the use of such techniques to improve patient function at a young age. Further research is needed to establish ambitious treatment objectives for the use of neuromuscular techniques in orthodontics.

\section{CONSENT}

Written informed consent was obtained from the patient and the patient's guardian for publication of this case report and accompanying images.

\section{ACKNOWLEDGEMENTS}

I gratefully acknowledge the patient and his family for their participation in the study. 


\section{REFERENCES}

1. Jankelson B. Neuromuscular aspects of occlusion. Effects of occlusal position on the physiology and dysfunction of the mandibular musculature. Dent Clin North Am. 1979;23(2):157-68.

2. Jankelson B. Measurement accuracy of the mandibular kinesiograph - a computerized study. J Prosthet Dent. 1980;44(6):656-66.

3. Jankelson B. Three-dimensional orthodontic diagnosis and treatment. A neuromuscular approach. J Clin Orthod. 1984;18(9):627-36.

4. Jankelson B. Physiology of human dental occlusion. J Am Dent Assoc. 1955;50(6):664-80.

5. Jankelson B, Hoffman GM, Hendron JA Jr. The physiology of the stomatognathic system. J Am Dent Assoc, 1952;46(4): 375-86.

6. Jankelson B, Swain CW, Crane PF, Radke JC. Kinesiometric instrumentation: a new technology. J Am Dent Assoc. 1975;90(4):834-40.

7. Neuromuscular dental diagnosis and treatment: Robert R. Jankelson Ishiyaku EuroAmerica, St. Louis: 1990. American Journal of Orthodontics and Dentofacial Orthopedics: official publication of the American Association of Orthodontists, its constituent societies, and the American Board of Orthodontics, 1991. 99(3): p. 283-284.

8. Hannam AG, De Cou RE, Scott JD, Wood WW. The relationship between dental occlusion, muscle activity and associated jaw movement in man. Arch Oral Biol. 1977;22(1):25-32.

9. Hannam AG, DeCou RE, Scott JD, Wood WW. The kinesiographic measurement of jaw displacement. J Prosthet Dent. 1980;44(1):88-93.

10. Hannam AG, Scott JD, De Cou RE. A computer-based system for the simultaneous measurement of muscle activity and jaw movement during mastication in man. Arch Oral Biol. 1977;22(1):17-23.

11. Cooper BC. The role of bioelectronic instrumentation in the documentation and management of temporomandibular disorders. Oral Surg Oral Med Oral Pathol Oral Radiol Endod. 1997;83(1):91-100.

12. Food and Drug Administration, HHS. Medical devices: classification of the dental sonography device and jaw tracking device. Final rule. Fed Regist. 2003 Dec 2;68(231):67365-7.

13. Cooper BC. The role of bioelectronic instruments in the management of TMD. N Y State Dent J. 1995;61(9):48-53.

14. Stohler C. Advanced investigative method for the study of natural mastication - a methodological report. SSO Schweiz Monatsschr Zahnheilkd. 1983;93(12):1189-96.

15. Maruyama T, Miyauchi S, Umekoji E. Analysis of the mandibular relationship of TMJ dysfunction patients using the Mandibular Kinesiograph. J Oral Rehabil. 1982;9(3):217-23.

16. Ahlgren J, Sonesson B, Blitz M. An electromyographic analysis of the temporalis function of normal occlusion. Am J Orthod. 1985;87(3):230-9.

17. Bakke M, Michler L, Möller E. Occlusal control of mandibular elevator muscles. Scand J Dent Res. 1992;100(5):284-91.

18. Blanksma NG, Van Eijden TM. Electromyographic heterogeneity in the human temporalis muscle. J Dent Res. 1990;69(10):1686-90.

19. Blanksma NG, van Eijden TM. Electromyographic heterogeneity in the human temporalis and masseter muscles during static biting, open/close excursions, and chewing. J Dent Res. 1995;74(6):1318-27.

20. Donegan SJ, Carr AB, Christensen LV, Ziebert GJ. An electromyographic study of aspects of 'deprogramming' of human jaw muscles. J Oral Rehabil. 1990 Nov;17(6):509-18.
21. Schindler HJ, Blaser W. [T.E.N.S., a myofunctional concept for oral rehabilitation]. Quintessenz. 1982;33(11):2181-91. German.

22. Schindler HJ, Blaser W, Rath E. [T.E.N.S. (transcutaneous electroneurostimulation), a myofunctional concept for oral rehabilitation (II)]. Quintessenz. 1982 Feb;33(2):295-307. German.

23. Konchak PA, Thomas NR, Lanigan DT, Devon RM. Freeway space measurement using mandibular kinesiograph and EMG before and after TENS. Angle Orthod. 1988;58(4):343-50.

24. Lerman MD. The muscle engram: the reflex that limits conventional occlusal treatment. Cranio. 2011;29(4):297-303.

25. Lerman MD. A revised view of the dynamics, physiology, and treatment of occlusion: a new paradigm. Cranio. 2004;22(1):50-63.

26. Konchak PA, Thomas NR, Lanigan DT, Devon R. Vertical dimension and freeway space. A kinesiographic study. Angle Orthod. 1987;57(2):145-54.

27. Dosumu OO, Ikusika OF. An assessment of interocclusal space in a dentate Nigerian population. Niger Postgrad Med J. 2013;20(4):315-8.

28. Johnson A, Wildgoose DG, Wood DJ.The determination of freeway space using two different methods. J Oral Rehabil. 2002;29(10):1010-3.

29. Tingey EM, Buschang PH, Throckmorton GS. Mandibular rest position: a reliable position influenced by head support and body posture. Am J Orthod Dentofacial Orthop. 2001;120(6):614-22.

30. Kamyszek G, Ketcham R, Garcia R Jr, Radke J. Electromyographic evidence of reduced muscle activity when ULF-TENS is applied to the Vth and VIIth cranial nerves. Cranio. 2001;19(3):162-8.

31. Sherry JE, Oehrlein KM, Hegge KS, Morgan BJ. Effect of burst-mode transcutaneous electrical nerve stimulation on peripheral vascular resistance. Phys Ther. 2001;81(6):1183-91.

32. Forrester SE, Allen SJ, Presswood RG, Toy AC, Pain MT. Neuromuscular function in healthy occlusion. J Oral Rehabil. 2010;37(9):663-9.

33. Hickman DM, Cramer R. The effect of different condylar positions on masticatory muscle electromyographic activity in humans. Oral Surg Oral Med Oral Pathol Oral Radiol Endod. 1998;85(1):18-23.

34. Hickman DM, Cramer R, Stauber WT. The effect of four jaw relations on electromyographic activity in human masticatory muscles. Arch Oral Biol. 1993;38(3):261-4.

35. Yamashita A, Kondo Y, Yamashita J. Thirty-year follow-up of a TMD case treated based on the neuromuscular concept. Cranio. 2014;32(3):224-34.

36. Manni A, Brunori P, Ursini R, Deli R. [Neuromuscular occlusion in the orthodontic treatment of craniomandibular disorders. A clinical case]. Minerva Stomatol. 2002;51(3):95-101. Italian.

37. Monaco A, Cattaneo R, Marci MC, Marzo G, Gatto R, Giannoni M. Neuromuscular diagnosis in orthodontics: effects of TENS on maxillomandibular relationship. Eur J Paediatr Dent. 2007 Sep;8(3):143-8.

38. Yamashita A, Kondo Y, Yamashita J. Thirty-year follow-up of a TMD case treated based on the neuromuscular concept. Cranio. 2014 Jul;32(3):224-34.

39. Manchanda S, Bhawsar SV, Manchanda J, Ramchandani A. Recording Myocentric Relation in a Partially Edentulous Patient: A Case Report. J Int Oral Health. 2015;7(Suppl 2):92-5. 